\title{
Fratura de fêmur em idosos no Piauí: um estudo retrospectivo de 2009 a 2019
}

Femur fracture in the elderly in Piauí: a retrospective study from 2009 to 2019

Fractura de fémur en ancianos en Piauí: un estudio retrospectivo de 2009 a 2019

Gideon Batista Viana Júnior ${ }^{1 *}$, Ivan Bruno da Silva Ferreira ${ }^{1}$, Allan Dellon da Silva ${ }^{1}$, Álvaro Francisco

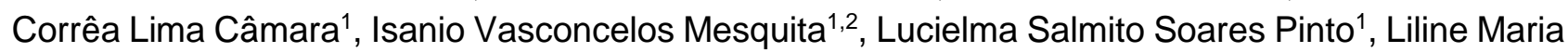
Soares Martins ${ }^{1}$, Luciana Tolstenko Nogueira ${ }^{1}$.

\section{RESUMO}

Objetivos: Descrever a evolução temporal da taxa de incidência de fraturas de fêmur em idosos e caracterizar o perfil dessas internações hospitalares e dos óbitos no Estado do Piauí. Métodos: Estudo descritivo, de corte transversal, com abordagem quantitativa sobre os dados das internações hospitalares e óbitos em idosos com mais de 60 anos diagnosticados com fratura de fêmur, no Estado do Piauí, no período de janeiro de 2009 a dezembro de 2019. A população de estudo refere-se às internações por fratura de fêmur contidas no Sistema de Informações Hospitalares - SIH/SUS. Resultados: O presente estudo evidenciou um total de 7023 internações por fratura de fêmur em idosos no Estado do Piauí. Desse total de internações, 2229 eram pessoas do sexo masculino e 4794 do sexo feminino, sendo que o maior número de internações ocorreu na faixa etária de 80 anos ou mais, totalizando 3389. No tocante ao número de óbitos, o Estado totalizou 281 mortes nessa população. Conclusão: Verificou-se números cada vez maiores de episódios de fratura de fêmur em idosos no Piauí.

Palavras-chave: Fraturas do fêmur, Hospitalização, Idoso.

\begin{abstract}
Objectives: To describe the temporal evolution of the incidence rate of femoral fractures in the elderly and to characterize the profile of these hospitalizations and deaths in the State of Piauí. Methods: Descriptive, crosssectional study, with a quantitative approach on the data of hospitalizations and deaths in elderly people over 60 years diagnosed with femur fracture, in the State of Piauí, from January 2009 to December 2019. The study population refers to femoral fracture hospitalizations contained in the Hospital Information System - HIS/SUS. Results: The present study showed that a total of 7,023 hospitalizations for femoral fracture in the state of Piauí. Of this total number of hospitalizations, 2,229 were male and 4,794 female, and the highest number of hospitalizations occurred in the age group of 80 years or more, totaling 3389. Regarding the number of deaths, the state totaled 281 deaths in this population. Conclusion: There were increasing numbers of femoral fracture episodes in the elderly in Piauí.
\end{abstract}

Keywords: Femoral fractures, Hospitalization, Old.

\section{RESUMEN}

Objetivos: Describir la evolución temporal de la tasa de incidencia de fracturas femorales en las personas mayores y caracterizar el perfil de estas hospitalizaciones y muertes en el Estado de Piauí. Métodos: Estudio descriptivo y transversal, con un enfoque cuantitativo sobre los datos de hospitalizaciones y muertes en personas mayores de 60 años diagnosticados con fractura de fémur, en el estado de Piauí, de enero de 2009

1 Universidade Estadual do Piauí (UESPI), Teresina - PI. *E-mail: gideon.viana.jr@gmail.com

${ }^{2}$ Hospital Getúlio Vargas (HGV), Teresina - PI. 
a diciembre de 2019. La población del estudio se refiere a hospitalizaciones por fractura femoral contenidas en el Sistema de Información Hospitalaria - SIH/SUS. Resultados: El presente estudio mostró un total de 7.023 hospitalizaciones por fractura femoral en ancianos del estado de Piauí. De este número total de hospitalizaciones, 2.229 fueron hombres y 4.794 mujeres, y el mayor número de hospitalizaciones se produjo en el grupo de edad de 80 años o más, con un total de 3389. En cuanto al número de muertes, el estado sumó 281 muertes en esta población. Conclusión: Hubo un número creciente de episodios de fractura femoral en los ancianos de Piauí.

Palabras clave: Fracturas femorales, Hospitalización, Viejo.

\section{INTRODUÇÃO}

De acordo com o Estatuto do Idoso, atualmente, no Brasil, define-se como pessoa idosa qualquer cidadão que possua idade igual ou superior a 60 anos (BRASIL, 1999; BRASIL, 2003). Por certo, o aumento da expectativa de vida é uma realidade que se tornou evidente, e fatores internos e externos podem estar relacionados ao aumento do número de idosos, contudo ainda existem muitos desafios quando se trata da assistência à saúde desse grupo populacional (MOREIRA RS, et al., 2021).

O Instituto Brasileiro de Geografia e Estatística (IBGE), com base em modelos de projeção, estimou, no ano de 2018 , que $13 \%$ da população brasileira se encontrava com 60 anos ou mais, correspondendo a aproximadamente 19,2 milhões de pessoas. Desse modo, estima-se que no ano de 2060 o Brasil terá uma população de idosos superior à de jovens (PETERLE VCU, et al., 2020).

Inevitavelmente, as mudanças que ocorrem no envelhecimento são naturais e podem levar a limitações tanto cognitivas quanto funcionais. Essas limitações podem vir associadas a afecções agudas ou crônicas, tornando essa população idosa mais vulnerável aos riscos que o ambiente pode oferecer, e como resultado poderá possivelmente aumentar a ocorrência de quedas (VIEIRA LS, et al., 2018).

Nesse ínterim, é importante citar que o evento queda aparece entre as causas que prioritariamente mais afetam a saúde da pessoa idosa. Da mesma forma, as quedas ocorrem pela combinação de vários fatores, dentre eles o ambiente no qual o idoso está inserido, visto que os ambientes com superfícies instáveis são um grande fator de risco, como por exemplo, pisos escorregadios e irregulares, pois requerem um maior controle do equilíbrio (REBÊLO FL, et al., 2020).

A fratura do fêmur em idosos geralmente ocorre por traumas de baixa energia e está relacionada a fatores como a idade avançada, fraqueza muscular, baixa ingestão de cálcio e vitamina $D$, diminuição da acuidade visual, abuso de álcool, uso de medicamentos psicotrópicos, redução funcional da capacidade de metabolizar medicações, desnutrição, geometria do quadril, predisposição genética e a osteoporose (OLIVEIRA CC e BORBA VZC, 2017).

A estabilização cirúrgica é uma das principais opções de escolha para o tratamento da fratura do fêmur. Porém, surpreendentemente, estudos demonstram que até $50 \%$ dos pacientes com fratura proximal do fêmur morrem nos primeiros seis meses após o trauma, e que muitos pacientes submetidos à cirurgia não recuperam sua função basal e independência (PETROS RSB, et al., 2017).

Dessa forma, a caracterização, assim como a compreensão do atual impacto da fratura de fêmur na população idosa brasileira, bem como no estado do Piauí e no município de Teresina pode auxiliar no planejamento de ações preventivas voltadas para a assistência médica desse agravo à saúde. Nesse sentido, o estudo tem como objetivo descrever a evolução temporal da taxa de incidência de fraturas de fêmur em idosos e caracterizar o perfil dessas internações hospitalares e dos óbitos no Estado do Piauí.

\section{MÉTODOS}

Trata-se de um estudo descritivo, de corte transversal, com abordagem quantitativa sobre os dados das internações hospitalares e óbitos em idosos com mais de 60 anos diagnosticados com fratura de fêmur, no Estado do Piauí, no período de janeiro de 2009 a dezembro de 2019. 
A população de estudo refere-se às internações por fratura de fêmur, sem discriminar a localização anatômica específica, observando o código CID-10 S72 (fratura de fêmur), ocorridas no período de 2009 a 2019, contidas no Sistema de Informações Hospitalares (SIH/SUS) e disponibilizadas pelo Departamento de Informática do Sistema Único de Saúde (DATASUS), que contém sistemas de informações em saúde disponíveis pela Internet, no website http://www2.datasus.gov.br.

As informações sobre morbidade hospitalar, disponíveis no DATASUS, são provenientes das Autorizações de Internação Hospitalar (AlH) preenchidas pelo médico responsável pela internação hospitalar e consolidadas pelas Secretarias de Saúde Municipais e dos Estados.

Os dados populacionais utilizados foram as estimativas (2009 a 2019) e os dados censitários (2010) do Instituto Brasileiro de Geografia e Estatística (IBGE), disponíveis no sítio eletrônico do DATASUS.

Foram coletados para o estudo, dados de perfil: sexo, faixa etária ( 60 a 69 anos, 70 a 79 anos e 80 anos ou mais), número de internações hospitalares e óbitos no âmbito Nacional, por Regiões Brasileiras e Macrorregiões de Saúde do Piauí.

Os dados foram organizados em planilhas do software Microsoft Excel 2019 e Microsoft Word 2019, sendo calculadas e organizadas as frequências absolutas e relativas de cada variável, havendo a distribuição dos dados por meio de gráficos e tabelas ao longo do capítulo de resultados.

A coleta de dados ocorreu no mês de outubro de 2020 por meio da utilização do programa Tabnet, que permitiu a tabulação das grandes massas de dados. Esse estudo obedeceu aos seguintes critérios de inclusão: sujeitos com 60 anos de idade ou mais e ocorrência de hospitalização entre os anos de 2009 e 2019. A apreciação e a aprovação pelo Comitê de Ética em Pesquisa desse estudo foi dispensada porque a pesquisa se baseou em dados disponibilizados em meio eletrônico pelo Ministério da Saúde, dados estes de domínio público.

\section{RESULTADOS}

No período de janeiro de 2009 a dezembro de 2019 foram registradas 7023 internações hospitalares por fratura de fêmur em idosos no Estado do Piauí, com maior taxa de incidência de internações no ano de 2017 (28,94/100000 habitantes) e menor incidência em 2010 (11,46/100000 habitantes), correspondendo a uma média de 638,45 internações para cada ano. Constatou-se que aproximadamente $68 \%$ das internações no Piauí eram pessoas do sexo feminino (Gráfico 1).

Gráfico 1 - Número de Internações Hospitalares por Fratura de Fêmur em Idosos, em relação ao sexo, no Piauí entre os anos de 2009 e 2019.

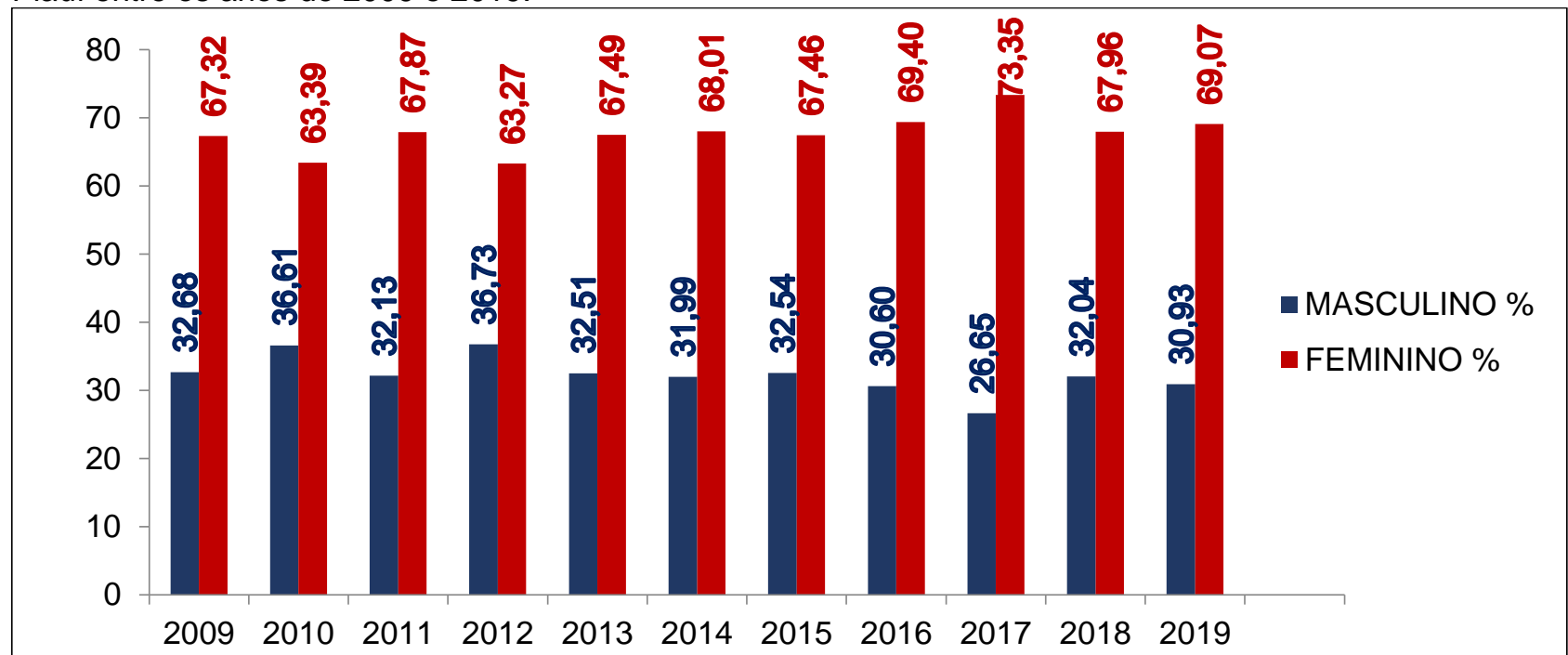

Fonte: Viana Júnior GB, et al., 2021; dados extraídos do site do Ministério da Saúde - Sistema de Informações Hospitalares do SUS (SIH/SUS). 
Verificou-se que a faixa etária de 80 anos ou mais foi a que teve maior número de internações, incluindo homens e mulheres, no Piauí, na Região Nordeste e no Brasil, durante o período estudado (Gráfico 2).

Gráfico 2 - Número de Internações Hospitalares por fratura de fêmur, em relação à faixa etária, no Brasil, Nordeste e Piauí entre os anos de 2009 - 2019.

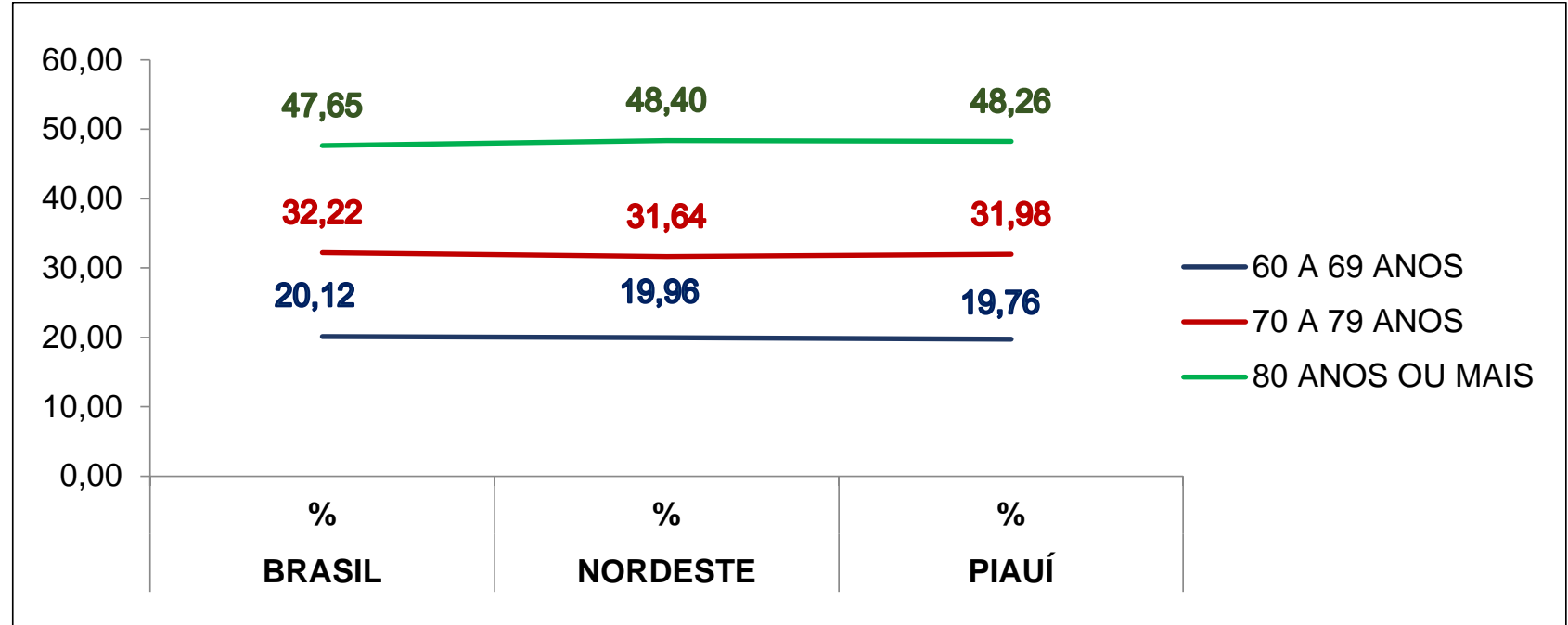

Fonte: Viana Júnior GB, et al., 2021; dados extraídos do site do Ministério da Saúde - Sistema de Informações Hospitalares do SUS (SIH/SUS).

Comparando a taxa de incidência das internações hospitalares por fratura de fêmur em idosos, o estado do Piauí no ano de 2009 apresentou uma taxa maior que a Região Nordeste e o Brasil, com 19,38 internações por 100000 habitantes. Já no ano de 2019, último ano do estudo, o Piauí com 24,10 internações por 100000 ainda manteve a taxa de internação maior que a Região Nordeste (21,4 internações por 100000 habitantes), porém, menor do que a taxa de internação hospitalar no Brasil (30,02 internações por 100000 habitantes) (Gráfico 3).

Gráfico 3 - Taxa de Incidência* das Internações Hospitalares por fratura de fêmur no Brasil, Nordeste, Piauí entre os anos $2009-2019$.

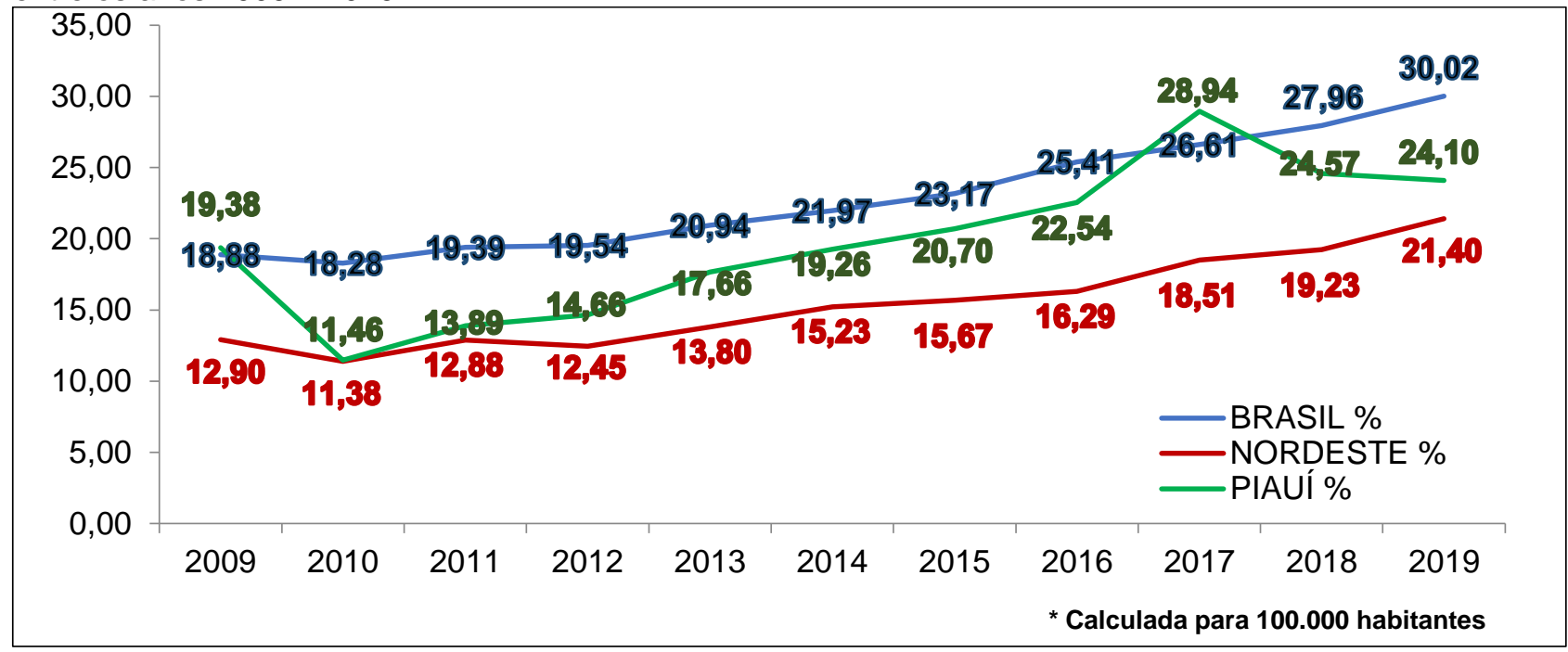

Fonte: Viana Júnior GB, et al., 2021; dados extraídos do site do Ministério da Saúde - Sistema de Informações Hospitalares do SUS (SIH/SUS).

Em relação à taxa de crescimento das internações hospitalares, o ano de 2017 foi o de maior crescimento em relação ao ano anterior, com 28,69\%, e o ano de 2010 foi o que teve a menor taxa de crescimento, com 
uma queda de 40,49\% em relação ao ano anterior, no Estado do Piauí. O Brasil, com exceção do ano de 2010, sempre manteve uma taxa de crescimento crescente. Já a Região Nordeste, assim como o Piauí, teve a sua maior taxa de crescimento das internações hospitalares por fatura de fêmur em idosos no ano de 2017, aumentando 14,19\% as internações em relação ao ano anterior (Gráfico 4).

Gráfico 4 - Taxa de Crescimento de Internações Hospitalares por fratura de fêmur no Brasil, Nordeste e Piauí ente os anos de $2009-2019$.

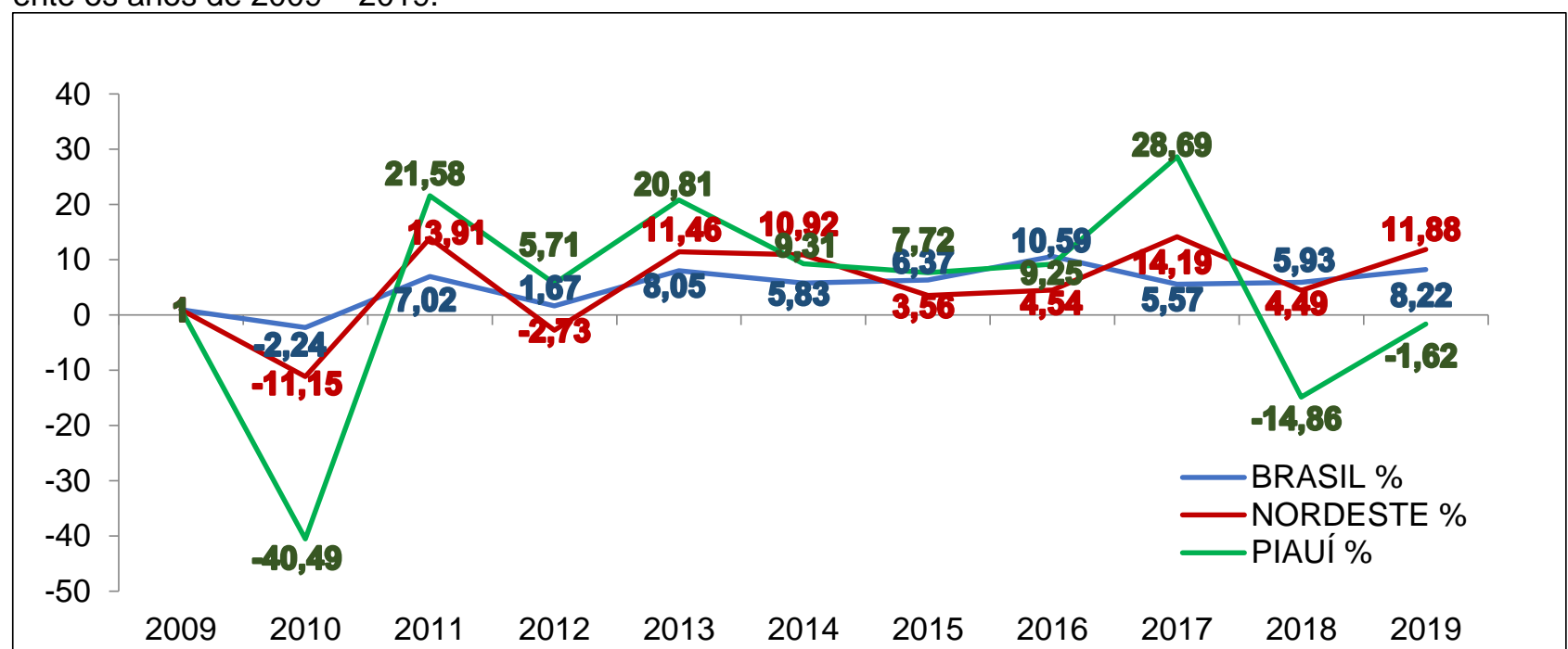

Fonte: Viana Júnior GB, et al., 2021; dados extraídos do site do Ministério da Saúde - Sistema de Informações Hospitalares do SUS (SIH/SUS).

No que se refere às Macrorregiões de Saúde do Estado do Piauí, a Macrorregião do Meio Norte foi a que em todos os anos teve o maior número de internações hospitalares por fratura de fêmur em idosos, totalizando 6240 internações, com uma maior taxa de incidência de 58,97 internações por 100000 habitantes (Tabela 1).

Tabela 1 - Taxa de Incidência das Internações Hospitalares por Fratura de Fêmur por Macrorregiões de Saúde entre os anos de $2009-2019$.

\begin{tabular}{ccccccccc}
\hline \multirow{2}{*}{ ANO } & \multicolumn{2}{c}{ SEMI-ARIDO } & \multicolumn{2}{c}{ MEIO NORTE } & \multicolumn{2}{c}{ LITORAL } & \multicolumn{2}{c}{ CERRADOS } \\
\cline { 2 - 9 } & $\mathbf{N}^{\circ}$ & $\%$ & $\mathbf{N}^{\circ}$ & $\%$ & $\mathbf{N}^{\circ}$ & $\%$ & $\mathbf{N}^{\circ}$ & $\%$ \\
\hline 2009 & 21 & 3,62 & 569 & 42,28 & 24 & 3,62 & 1 & 0,17 \\
2010 & 16 & 2,75 & 319 & 23,53 & 30 & 4,51 & 1 & 0,17 \\
2011 & 18 & 3,09 & 398 & 29,25 & 29 & 4,34 & 0 & 0 \\
2012 & 35 & 5,99 & 420 & 30,76 & 15 & 2,24 & 1 & 0,17 \\
2013 & 16 & 2,73 & 539 & 39,36 & 11 & 1,64 & 3 & 0,5 \\
2014 & 23 & 3,92 & 579 & 42,15 & 19 & 2,82 & 1 & 0,17 \\
2015 & 27 & 4,59 & 590 & 42,8 & 51 & 7,55 & 2 & 0,33 \\
2016 & 18 & 3,06 & 643 & 46,5 & 69 & 10,2 & 2 & 0,33 \\
2017 & 15 & 2,54 & 818 & 58,97 & 94 & 13,86 & 15 & 2,5 \\
2018 & 20 & 3,38 & 697 & 50,08 & 78 & 11,47 & 7 & 1,16 \\
2019 & 28 & 4,73 & 668 & 47,84 & 66 & 9,68 & 27 & 4,48 \\
\hline TOTAL & $\mathbf{2 3 7}$ & & $\mathbf{6 2 4 0}$ & & $\mathbf{4 8 6}$ & & $\mathbf{6 0}$ &
\end{tabular}

Fonte: Viana Júnior GB, et al., 2021; dados extraídos do site do Ministério da Saúde - Sistema de Informações Hospitalares do SUS (SIH/SUS).

Das 7023 internações por fratura de fêmur em idosos que ocorreram no Piauí, 281 evoluíram para o óbito, o que demonstra uma taxa de letalidade de $4 \%$, no período em estudo. A taxa de letalidade do estado do Piauí foi maior que a registrada na Região Nordeste (3,63\%), porém menor que a do Brasil $(5,06 \%$ ) (Tabela 2). 
Tabela 2 - Taxa de Letalidade por Fratura de Fêmur no Brasil, Nordeste e Piauí entre 2009 - 2019.

\begin{tabular}{cccccccccc}
\hline \multirow{2}{*}{ ANO } & \multicolumn{3}{c}{ BRASIL } & \multicolumn{3}{c}{ NORDESTE } & \multicolumn{3}{c}{ PIAUÍ } \\
\cline { 2 - 10 } & Internações & Óbitos & $\%$ & Internações & Óbitos & $\%$ & Internações & Óbitos & $\%$ \\
\hline 2009 & 36.435 & 1.742 & 4,78 & 6.943 & 251 & 3,62 & 615 & 10 & 1,63 \\
2010 & 35.620 & 1.629 & 4,57 & 6.169 & 193 & 3,13 & 366 & 7 & 1,91 \\
2011 & 38.119 & 1.867 & 4,9 & 7.027 & 283 & 4,03 & 445 & 18 & 4,04 \\
2012 & 38.755 & 1.913 & 4,94 & 6.835 & 280 & 4,1 & 471 & 26 & 5,52 \\
2013 & 41.874 & 2.113 & 5,05 & 7.618 & 263 & 3,45 & 569 & 23 & 4,04 \\
2014 & 44.316 & 2.203 & 4,97 & 8.450 & 300 & 3,55 & 622 & 43 & 6,91 \\
2015 & 47.138 & 2.404 & 5,1 & 8.751 & 304 & 3,47 & 670 & 21 & 3,13 \\
2016 & 52.130 & 2.736 & 5,25 & 9.148 & 312 & 3,41 & 732 & 21 & 2,87 \\
2017 & 55.034 & 2.913 & 5,29 & 10.446 & 386 & 3,7 & 942 & 44 & 4,67 \\
2018 & 58.298 & 3.054 & 5,24 & 10.915 & 409 & 3,75 & 802 & 38 & 4,74 \\
2019 & 63.089 & 3.268 & 5,18 & 12.212 & 450 & 3,68 & 789 & 30 & 3,8 \\
\hline TOTAL & $\mathbf{5 1 0 8 0 8}$ & $\mathbf{2 5 8 4 2}$ & $\mathbf{5 , 0 6}$ & $\mathbf{9 4 5 1 4}$ & $\mathbf{3 4 3 1}$ & $\mathbf{3 , 6 3}$ & $\mathbf{7 0 2 3}$ & $\mathbf{2 8 1}$ & $\mathbf{4}$
\end{tabular}

Fonte: Viana Júnior GB, et al., 2021; dados extraídos do site do Ministério da Saúde - Sistema de Informações Hospitalares do SUS (SIH/SUS).

\section{DISCUSSÃO}

O presente estudo evidenciou que no SIH/SUS foi registrado um total de 7023 internações por fratura de fêmur em idosos no Estado do Piauí, durante o período de janeiro de 2009 a dezembro de 2019. Desse total de internações, 2229 eram pessoas do sexo masculino e 4794 do sexo feminino, sendo que o maior número de internações ocorreu na faixa etária de 80 anos ou mais, totalizando 3389. No tocante ao número de óbitos, o Estado totalizou 281 mortes nessa população, tendo uma taxa de letalidade de 4,0\%, assim sendo, 97 homens e 184 mulheres, com uma taxa de letalidade de 4,35 entre a população senil masculina e de 3,84 entre a feminina.

Neste intervalo de estudo, foi observado no Piauí que a incidência das internações hospitalares por fratura de fêmur em pessoas com idade igual ou superior a 60 anos, foi aproximadamente duas vezes maior em mulheres, em relação a dos homens (Gráfico 1). Esse padrão estatístico mostra-se concordante com os registros nacionais, que indicam prevalência do sexo feminino no total de hospitalizações em período semelhante ao estudado (68\% de prevalência entre 2008-2018) (PETERLE VCU, et al., 2020).

Na literatura podem ser citados múltiplos fatores para justificar essa tendência. Vieira LS, et al. (2018) cita por exemplo, a diferença da composição corporal entre os sexos. Mulheres possuem massa magra e força muscular em menor quantidade se comparada a homens.

Ainda em relação às diferenças de composição corporal, segundo Franco LG, et al. (2016), o principal mecanismo para o trauma de fêmur em idosos é o de baixa energia, ou seja, queda da própria altura, muito associada à osteoporose. Inclusive, muitos autores consideram esta doença crônica degenerativa como o principal fator de risco para fraturas ósseas em idosos, especialmente as que acometem ossos longos, como o fêmur (SANTOS NETO AAD, et al., 2017).

$\mathrm{O}$ aspecto social e familiar também é um fator importante a ser considerado. Dentre os mais velhos, as mulheres despontam como as principais vítimas de abuso (64\%), especialmente em relação à violência física e psicológica. Frequentemente, há associação ou relação de proximidade e até de parentesco entre 0 agressor e a vítima idosa, com tendência de que a violência ocorra em ambiente residencial (SOUZA TA, et al., 2020).

Apesar da prevalência em morbidade associada ao sexo feminino, Peterle VCU, et al. (2020) relata que a taxa de mortalidade e o número de dias de internação é ligeiramente maior no sexo masculino, independente da faixa etária, em pessoas maiores de 60 anos com fratura de fêmur. A razão média de mortalidade homem/mulher é de 1,13/1, mas a discrepância tende a ficar maior com o aumento da faixa etária considerada. 
Em um estudo sobre mortalidade em pacientes hospitalizados devido ao trauma em questão, Correa JGL, et al. (2020) também identificou o sexo masculino como um importante fator de risco, mencionando a hipótese de um maior número de comorbidades não diagnosticadas ou não tratadas corretamente, levando a um maior número de complicações severas em pós-operatório.

Ao observar o Gráfico 2, é inegável que o Piauí segue a tendência apresentada no Nordeste e no Brasil em relação ao número de internações por faixa etária, com o grupo de maiores de 80 anos representando quase $50 \%$ dos pacientes no período estudado. A idade representa um importante fator de risco para quedas ou fraturas em população idosa, com acréscimo de $8 \%$ no risco de chance de ocorrer uma fratura osteoporótica a cada ano de vida acrescido, a partir dos 45 anos (XAVIER RM, et al., 2019).

Essa incidência progressiva com o aumento da idade ocorre principalmente devido ao processo natural do envelhecimento, que através de alterações funcionais e estruturais do organismo, leva à redução da capacidade do sistema sensorial, força muscular, estabilidade e dinâmica das articulações, entre outras consequências (VIEIRA LS, et al., 2018). Dessa forma, o idoso fica cada vez mais dependente, com prejuízo no equilíbrio, mobilidade, flexibilidade e marcha (SANTOS NETO AAD, et al., 2017).

Outro fator relacionado a isso é o acúmulo de comorbidades. Quanto mais avançada a idade, ao mesmo tempo, é maior o risco de queda e a prevalência de múltiplas comorbidades (MATA LRF, et al., 2017). O estudo realizado por Moraes AS, et al. (2017), identificou em idosos que caíam de forma recorrente um aumento significativo no número de comorbidades (4 ou até mais), como artrite, osteoporose e tontura. Concomitantemente, a ocorrência de duas ou mais quedas teve prevalência significativamente maior em relação a episódio único de queda, em idosos com mais de 80 anos.

Dentre as doenças crônicas mais associadas aos mais velhos vítimas de queda, estão a osteoporose, hipertensão e diabetes mellitus. Pacientes hipertensos têm oito vezes mais risco de cair do que normotensos. Já a neuropatia periférica causada por diabetes mal controlado está associada à piora do desempenho de marcha e equilíbrio (CANUTO CPAS, et al., 2020).

Esse dado torna-se ainda mais crítico ao se considerar que tais doenças, com associação tão relevantes a episódios de queda e fratura em maiores de 60 anos poderiam ser evitadas ou controladas com medidas de caráter preventivo, como atenção básica em saúde de qualidade, prática de exercícios físicos regulares e mudança de hábitos alimentares (MACHADO WD, et al., 2017).

Ao se levar em conta o aspecto nutricional, no estudo realizado por Xavier RM, et al. (2019), ele identificou a suplementação de vitamina $D$ como um fator importante na prevenção de fratura osteoporótica, com uma redução de $90 \%$ do risco para esse evento. Contudo, Santos TAB, et al. (2021) afirmou que não conseguiu evidenciar influência dos níveis de vitamina $D$ sérico sobre os níveis de Osteoprotegerina/RANKL intraósseo de pacientes submetidos ao procedimento de implante endósseo.

Em contrapartida, o estudo de Guerra MTE, et al. (2016), identificou a associação significativa entre o risco de fratura do fêmur em idosos e a hipovitaminose de vitamina $D_{3}$. Igualmente Brito $G M$, et al. (2016) constatou que valores insuficientes de cálcio estavam associados a uma elevada deficiência de vitamina $D$.

Essa informação pode ajudar a compreender os motivos relacionados aos valores encontrados nesse estudo relativos às menores taxas de incidência de internações hospitalares (Gráfico 3) e da taxa de letalidade (Tabela 2) por fratura de fêmur no Piauí e Nordeste em comparação com a média brasileira no período estudado. Afinal, a população residente no estado do Piauí está exposta a muita luminosidade solar e de acordo com Okan F, et al. (2021) sessões de banho de sol previnem a deficiência de vitamina D. Essa constatação também foi evidenciada por Pereira-Santos M, et al. (2018) em seu estudo onde demonstrou que as maiores prevalências de deficiências de Vitamina D foram observadas nas regiões Sul e Suldeste do Brasil.

O estudo em tela mostrou-se concordante com a literatura no que se refere à mudança do perfil etário da população brasileira, evidenciando um aumento da população idosa e, consequentemente, uma elevação no número de casos de internação de idosos por fratura de fêmur. O Piauí, se comparado à Região Nordeste e ao Brasil, neste aspecto, destacou-se por obter um decréscimo considerável em três períodos. Por outro lado, em sua maior parte a taxa de crescimento foi superior em comparação com a taxa de decréscimo (Gráfico 4). 
No que tange ao fato do Estado do Piauí possuir uma taxa de crescimento superior, na maioria dos anos estudados, se comparado à Região Nordeste e ao Brasil (Gráfico 4), Cortez ACL, et al. (2018) evidenciam que a falta de projetos sociais, a não criação de trabalhos multiprofissionais, assim como o não comprometimento desses profissionais pelo interesse na qualidade de vida dessa população, influenciam negativamente à retirada desses indivíduos da inatividade física e da depressão, elementos estes que são aliados da perda de funcionalidade do idoso, contribuindo para que ocorra traumas com fraturas, incluindo as de fêmur.

Dessa forma, é imprescindível uma sensibilização dos gestores de saúde, pois, assim como evidenciado no estudo de Santos TRS, et al. (2021) é importante priorizar o cuidado do ser humano do ponto de vista integral, considerando que a educação em saúde é uma boa estratégia a ser trabalhada com a população idosa, e dessa forma a equipe de saúde deve também objetivar o bem-estar dessa população.

Soares DS, et al. (2015) apontam para alguns dos principais fatores relacionados ao aumento do índice de quedas e fraturas de fêmur em idosos, como a presença de doenças crônicas, sedentarismo e consequente declínio da independência funcional, bem como a adesão às polifarmárcias, que é muito comum nessa fase, associado a isso, o ambiente domiciliar, que é comumente o local mais frequente das quedas, a depender das condições, pode oferecer ameaça, visto que a presença de escada sem corrimão, e de superfícies irregulares e escorregadias são fatores de alto risco para a ocorrência de quedas.

Já o estudo de Moura RB, et al. (2017), que investigou a percepção ao risco de queda feita com idosos que moram na capital do estado do Piauí, observaram que muitas queixas estavam relacionadas à estrutura do ambiente externo às casas, como a presença de buracos nas ruas e nas calçadas, a altura destas e a grande presença de degraus. Outra questão seria a carência de campanhas e de ações programáticas e estratégicas que visem à prevenção do medo de quedas recorrentes, o que acarreta em um baixo convívio social e a uma má qualidade de vida.

Quanto ao número de internações por Macrorregiões de Saúde no Piauí, constatou-se que a Macrorregião do Meio Norte foi a que teve o maior número de internações por fratura de fêmur em idosos no decorrer do período de estudo (Tabela 1). Esse resultado pode estar relacionado ao fato dessa região contemplar a capital do Estado, sendo dessa forma o centro de referência para atendimento de casos mais complexos. Assim como Mercenas SLG e Ferreira TPO (2019) ressaltaram em seu estudo, que o grande número de internações em somente uma região pode ser explicado por nela se concentrar o maior número de habitantes além de possuir o maior hospital público.

No que diz respeito à taxa de letalidade relacionada às fraturas de fêmur em idosos, o Piauí apresentou um valor abaixo do nacional, apesar de ser superior ao da Região Nordeste (Tabela 2). Uma pesquisa realizada por Peterle VCU, et al. (2020), revelou que o Nordeste foi a Região que apresentou a menor taxa de mortalidade por esse agravo.

Provavelmente esse resultado pode estar relacionado ao fato do nordestino estar mais exposto ao sol e, possivelmente devido a isso, apresentar boas taxas de vitamina $D$ em seu organismo. Ao se realizar um paralelo invertido com essa tese, Oliveira CA, et al. (2020) descreveu que a deficiência e a insuficiência de vitamina $D$ têm associação a distúrbios esqueléticos, bem como, observou que níveis baixos de vitamina $D$ podem estar ligados a um maior risco para a ocorrência de mialgias.

Ademais, Pereira TS, et al. (2020) relata que a Vitamina D possui extrema relevância na prática clínica e que é possível utilizá-la também como terapia menos invasiva para prevenir doenças de grande prevalência na população, pois é simples a sua obtenção em níveis séricos adequados. Analogamente, Queiroz MRP (2021) mostrou que a Vitamina $D$ tem efeito benéfico especialmente na população idosa.

No que concerne à taxa de letalidade ser maior no Estado do Piauí em relação à região Nordeste (Tabela 2), Franco LG, et al. (2016) explanam em seu trabalho que existem algumas explicações para este achado. Como as muitas complicações pós-cirúrgicas (infecções, tromboembolismo pulmonar, embolia gordurosa), acompanhadas de um longo processo de postergação de procedimentos cirúrgicos, seja por falta de vaga em Unidades de Terapia Intensiva (UTI) ou condições clínicas adequadas dos pacientes. Corroborando com as 
afirmações anteriores, Pinto IP, et al. (2019) descreveu que em pacientes com mais de 60 anos, compensados clinicamente, existe um aumento na mortalidade de indivíduos previamente hígidos quando o procedimento cirúrgico é realizado após 48 horas.

\section{CONCLUSÃO}

O País passa por um processo de envelhecimento da população, o que traz como consequência números cada vez maiores de episódios de fratura de fêmur em idosos. Assim, observou-se que o Piauí acompanhou essa tendência nacional. Somado a isso, o acúmulo de comorbidades entre os mais velhos, a falta de conhecimento da população na prevenção contra queda de idosos, a concentração da rede médica-hospitalar do estado na capital e em outras regiões do Estado do Piauí, bem como a falta de infraestrutura adequada desta para suportar essa quantidade de vítimas desse trauma e tratá-lo adequadamente, contribuem para que este problema seja ainda mais oneroso à saúde pública piauiense. Espera-se que esse estudo contribua para nortear futuras ações de prevenção e intervenção referentes a esse problema.

\section{REFERÊNCIAS}

1. BRASIL. Gabinete do Ministro de Estado da Saúde (BR). Portaria № 1.395 de 9 de dezembro de 1999: aprova a Política Nacional de Saúde do Idoso e dá outras providências. Diário Oficial [da] República Federativa do Brasil, 13 dez. 1999. Acesso em: 15 dez. 2020.

2. BRASIL. Lei $n^{\circ} 10.741$, de $1^{\circ}$ de outubro de 2003. Dispõe sobre o Estatuto do Idoso e dá outras providências. Diário Oficial da União, 3 dez. 2003a. Disponível em: <http://www.planalto.gov.br/ccivil_03/decreto/d1948.htm>. Acesso em: 15 dez. 2020.

3. BRITO GM, et al. Nutrientes e parâmetros bioquímicos relacionados com a saúde óssea em mulheres maior do que 65 anos. Acta bioquím. clín. Latinoam, 2016; 50 (3).

4. CANUTO CPAS, et al. Segurança do paciente idoso hospitalizado: uma análise do risco de quedas. Revista da Escola de Enfermagem da Universidade de São Paulo, 2020; 54: 1-9.

5. CORREA JGL, et al. Evaluation of predictive factors of in hospital mortality in patients with proximal femoral fracture. Acta Ortopédica Brasileira, 2020; 28: 40-43.

6. CORTEZ ACL, et al. Correlation among the Tests of Functional Capacity Evaluation of Elderly Participants of a Social Inclusion Project in the City of Teresina Piauí. Journal Of Health Sciences, 2018; 20: 277-282.

7. FRANCO LG, et al. Fatores associados à mortalidade em idosos hospitalizados por fraturas de fêmur. Rev. bras. Ortop., 2016; 51: 509-514.

8. GUERRA MTE, et al. Idosos com fratura da extremidade proximal do fêmur apresentam níveis significativamente menores de 25-hidroxivitamina D. Sociedade Brasileira de Ortopedia e Traumatologia, 2016; 51: 583-588.

9. MACHADO WD, et al. Elderly with not transmitted chronic diseases: a group association study. Revista Ciências e Saberes, 2017; 3: 444-451.

10. MATA LRF, et al. Fatores associados ao risco de queda em adultos no pós-operatório: estudo transversal. Revista Latino-Americana de Enfermagem, 2017; 25: e2904.

11. MERCENAS SSG, FERREIRA TPO. Caracterização das autorizações de internações hospitalares de idosos em Sergipe: um estudo ecológico do ano de 2018. TCC (Graduação em Enfermagem) - Universidade Tiradentes, Aracajú, 2019; 16 p.

12. MORAES AS, et al. Características das quedas em idosos que vivem na comunidade: estudo de base populacional. Revista Brasileira de Geriatria e Gerontologia, 2017; 20: 693-704.

13. MOREIRA RS, et al. Mortalidade em idosos com fratura de fêmur proximal em um hospital universitário. Revista Eletrônica Acervo Saúde, 2021; 13(1): e6382.

14. MOURA RB, et al. Percepção de idosos sobre o risco de queda. Revista Interdisciplinar, 2017; 10: 1-13.

15. OKAN F, et al. The Effect of Sun Light Exposure to the Level of Vitamin D in Elderly People Living in Nursing Home. Journal of Clinical Densitometry: Assessment \& Management of Musculoskeletal Health, 2021:1-11.

16. OLIVEIRA CA, et al. Análise da correlação entre hipovitaminose D, dor crônica e depressão em idosos. Revista Eletrônica Acervo Saúde, 2020; 12(12): e4738.

17. OLIVEIRA CC, BORBA VZC. Epidemiology of femur fractures in the elderly and cost to the state of Paraná, Brazil. Acta ortop. bras., 2017; 25: 155-158.

18. PEREIRA-SANTOS M, et al. Epidemiology of vitamin $D$ insufficiency and deficiency in a population in a sunny country: Geospatial meta-analysis in Brazil. Critical reviews in food science and nutrition. 2018; 59(13).

19. PEREIRA TS, et al. Avaliação da reposição semanal de vitamina $D$ em adultos e idosos não-obesos. Revista Eletrônica Acervo Científico, 2020; 12: e4989. 
20. PETERLE VCU, et al. Indicators of morbidity and mortality by femur fractures in older people: a decade-long study in brazilian hospitals. Acta ortop. bras., 2020; 28: 142-148.

21. PETROS RSB, et al. Influence of proximal femur fractures in the autonomy and mortality of elderly patients submitted to osteosynthesis with cephalomedullary nail. Rev. bras. ortop., 2017; 52: 57-62.

22. PINTO IP, et al. A cirurgia precoce nas fraturas do fêmur proximal em idosos reduz a taxa de mortalidade? Revista Brasileira de Ortopedia, 2019; 54: 392-395.

23. QUEIROZ MRP, et al. Estudo piloto sobre possível influência das vitaminas $D$ e $B$ nas dislipidemias: alvos para a medicina preventiva. Revista Eletrônica Acervo Saúde, 2021; 13(5): e7201.

24. REBÊLO FL, et al. Preocupação em relação à queda em idosos participantes de um programa de prevenção em um município do nordeste brasileiro. Revista Eletrônica Acervo Científico, 2020; 17: e5490.

25. SANTOS NETO AAD, et al. Fratura de fêmur em idosos hospitalizados: revisão integrativa. Cadernos de Graduação - Ciências Biológicas e da Saúde - UNIT, 2017; 4: 203-214.

26. SANTOS TAB, et al. Análise da correlação entre os níveis séricos da vitamina $D$ e os níveis de OPG/RANKL intraósseo. Revista Eletrônica Acervo Saúde, 2021; 13(2): e6063.

27. SANTOS TRS, et al. Ações virtuais de educação em saúde para população idosa: uma intervenção na comunidade. Revista Eletrônica Acervo Saúde, 2021; 13(6): e7467.

28. SOARES DS, et al. Análise dos fatores associados a quedas com fratura de fêmur em idosos: um estudo caso-controle. Revista Brasileira de Geriatria e Gerontologia, 2015; 18: 239-248.

29. SOUZA TA, et al. Plano de ação para o enfrentamento da violência contra a pessoa idosa no Brasil: análise dos indicadores por Unidades Federativas. Revista Brasileira de Geriatria e Gerontologia, 2020; 23: e200106.

30. VIEIRA LS, et al. Quedas em idosos no Sul do Brasil: prevalência e determinantes. Rev. Saúde Públi, $2018 ; 52: 1$-13.

31. XAVIER RM, et al. Profile of patients with osteoporotic fractures and factors that decrease prevention. Acta Ortopédica Brasileira, 2019; 27: 95-99. 